\title{
Alcoholic Gas Discrimination Using 32-Channel Fiber-Optic Sensor Array
}

\author{
Shinzo Yamakawa \\ (Toyama National College of Maritime Technology)
}

\begin{abstract}
This paper describes the alcoholic gas discrimination experiment using an artificial neural network pattern recognition technique and dye absorbance spectra patterns from a 32-channel fiber-optic sensor array with different dye coatings, whose absorption spectra change with alcoholic gas exposure. The 32-channel absorption spectra were simultaneously measured by using 13 band-pass filters and 32 photodiodes, and data-processed by the pattern recognition method. The gas recognition system has been successfully applied to discrimination of alcoholic gases having similar chemical sutucture and found to discriminate methanol, ethanol, 1-propanol, and 2-propanol.
\end{abstract}

Keywords: 32-channel fiber-optic sensor array, dye absorbance spectrum pattern, artificial neural network, pattern recognition, alcohol ic gas discrimination

\section{Introduction}

The use of a sensor array in conjunction with pattern recognition techniques is essential for realizing an artificial odor or gas sensor such as olfactory organs with both high sensitivity and selectivity ${ }^{(1)}$, (2). We have recently proposed a new odor or gas recognition system using a 6-channel (6-ch) plastic optical fiber (POF) sensor array that have six different dye/ silicone coatings ${ }^{(3)}$, whose dye absorptions change with organic gas exposure. A data set of six dye absorbances at a particular wavelength point from the six different sensors was used as a set of valiables for pattern recognition, or was used as the input data for artificial neural network (ANN).

In the cources of development of pattern recognition techniques for molecular recognition in water and in air, we also proposed the use of a set or a pattern of six dye absorbance spectra over a wide wavelength range ${ }^{(4)}$ instead of the use of a set or a pattern of six absorbance data at a particular wavelength ( 6 inputs for ANN ${ }^{(3)}$. The six absorbance spectra from six POF sensors pere simultaneously measured by changing the band-pass filter ( 13 wavelength points over $400 \sim 700 \mathrm{~nm}$ at $25 \mathrm{~nm}$-interval, $6 \times 13$
$=78$ inputs for ANN). The increase in ANN input from 6 to 78 contributed to an improvement in the recognition rate of organic gases.

This paper describes the alcohol gas discrimination experiments using ANN pattern recognition technique and a set of 32 dye absorbance spectra from a 32 -ch POF sensor array with six different dye coatings. Accordingly, the sensor array has $5 \sim 6$ sensors with the same kind of dye, though the $5 \sim 6$ sensors have the dye concentration different from each other (thus they have different gas sensitivities). The $32 \mathrm{ab}-$ sorbance spectra (13 wavelength points over 400 $\sim 700 \mathrm{~nm}$ at $25 \mathrm{~nm}$-interval) were data-processed by the ANN pattern recognition method $(32 \times 13$ $=416$ inputs for $\mathrm{ANN}$ ).

The olfactory mucosa of mammal noses has ca. a thousand kinds of receptor proteins and some ten millions of olfactory cells. Thus, It has some ten thousands of olfactory cells with the same receptor protein ${ }^{(5)}$. The large number of olfactory cells with the same receptor protein contribute to the discremination of odor molecules $^{(5)}$. In this work, the increase in ANN input from 78 to 418 may contribute to an improvement in the recognition rate of alcohol gases having similar chemical structure as well as in the case of the olfactory sense of mamnal. 


\section{Experimental}

\subsection{Six-Channel Fiber-Optic Sensor Array}

The POF sensors with six different dye/silicone coatings were prepared by a method similar to that previously described (3). (4). The dyes used were NKX736, NK2684, NK2560, rhodamine B, NK2273, and NK2936. The NK-type dyes were purchased from Nippon Kankoh-Shikiso Kenkyusyo Inc. A jacketed POF, SH4001. Mitubishi Rayon Inc., was used for the sensor. The fiber and the jacket diameters were $1.0 \mathrm{~mm}$ and $2.2 \mathrm{~mm}$. The tips of two POFs with a smooth edge were dipped in a dye/silicone/ethanol solution to coat the fiber edge with a dye/silicone coating. The two coated fiber edges were fixed with an acrylic jig to form a 32-ch transmission-absorption-type POF sensor array as shown in Fig. 1.

\section{2 Dye Absorption Spectrum Measurement}

Figure 2 illustrates the experimental setup for 32 -ch spectrum measurement. The 32 spectra from a 32 -ch sensor array were simultaneously measured by changing the band-pass filter. The 13 different band pass filters of 400 to $700 \mathrm{~nm}$ at 25-nm interval were used to obtain the spec tra data or the spectrophotometric data at particular wavelengths instead of a monochronator used in the previous 6 -ch sensor system ${ }^{(3)}$.

In Fig. 2, light from a halogen lamp passed through a heat absorbing filter, a diffuser, and a band pass filter. The monochromated light entered the $32 \mathrm{POF}$ at the same time and reached the corresponding 32 sensors. The 32 light signals were absorbed during travel through the corresponding 32 dye coatings and were detected by 32 photodiodes via 32 POFs. The signals, including dye absorbance data, were fed to a computer via 32 current-voltage converters and a 32-ch A-D converter. By off-1ine processing of the stored spectra data, the calculation of $a b-$ sorbance $\Delta A=\log \left(V_{0} / V\right)$ (eq. 1 shown below) and the pattern recognition has been carried out.

\section{3 Gas Discrimination Experiment}

Hethano1, ethanol, 1-propanol, and 2-propanol were used for gas discrimination experiment. The change in dye absorbance spectrum with gas exposure were measured by using the same gas-

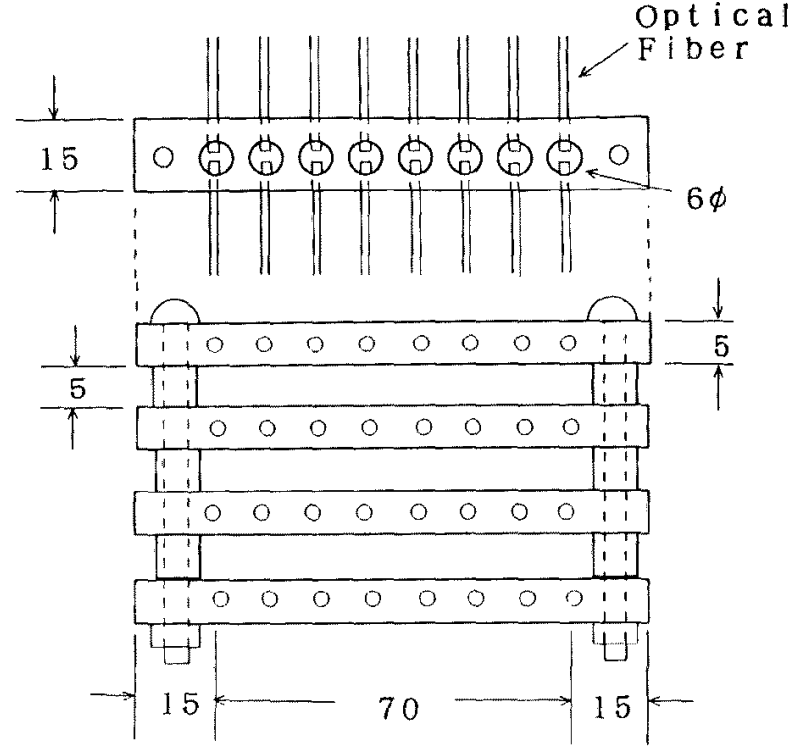

Fig. 1 Fiber-optic sensor array structure.

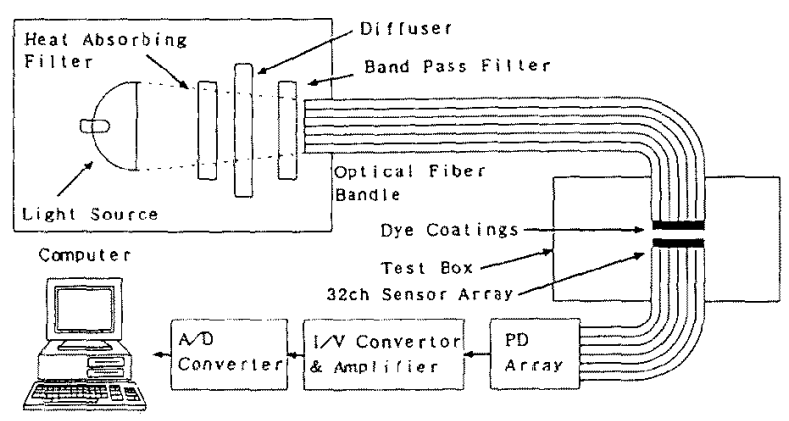

Fig. 2 Multichannel spectra measurement system.

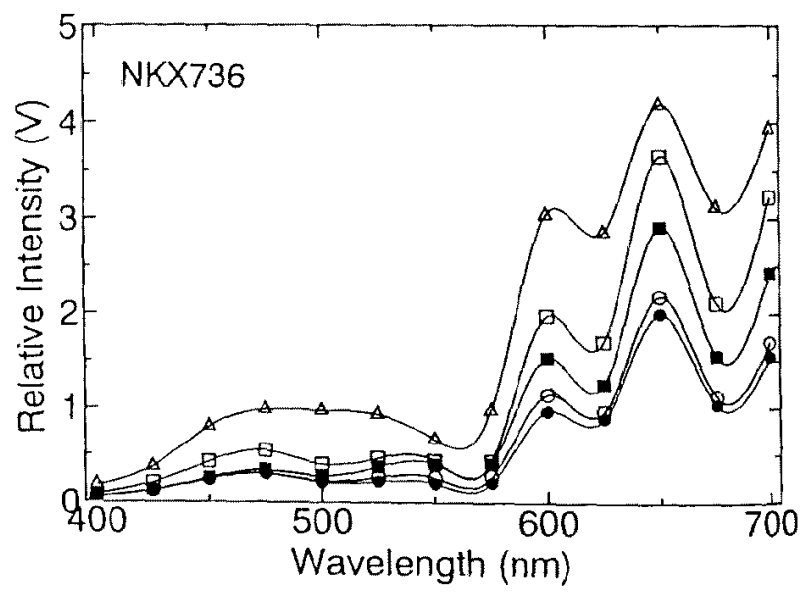

Fig. 3 Spectrum data from five POF sensors.

test box as used in the previous work ${ }^{(3),(4),(6)}$ under the saturated vapor pressure of alcohols. The absorbance $\Delta A=A-A_{0}$ is shown as follows. 


$$
\begin{aligned}
\Delta A & =A-A_{0}=\log \left(V_{F} / V\right)-\log \left(V_{F} / V_{0}\right) \\
& =\log \left(V_{0} / V\right)=k C
\end{aligned}
$$

Here, $V$ and $V_{0}$ are the input voltages to a $A / D$ converter from a dye-coated sensor in gas and in air, $V_{F}$ is the input voltage from an uncoated sensor in air, $C$ is the gas concentration, and $k$ is constant. The absorbance $\Delta A=\log \left(V_{0} / V\right)$ at each wavelength was used as the variable or as the input data for ANN.

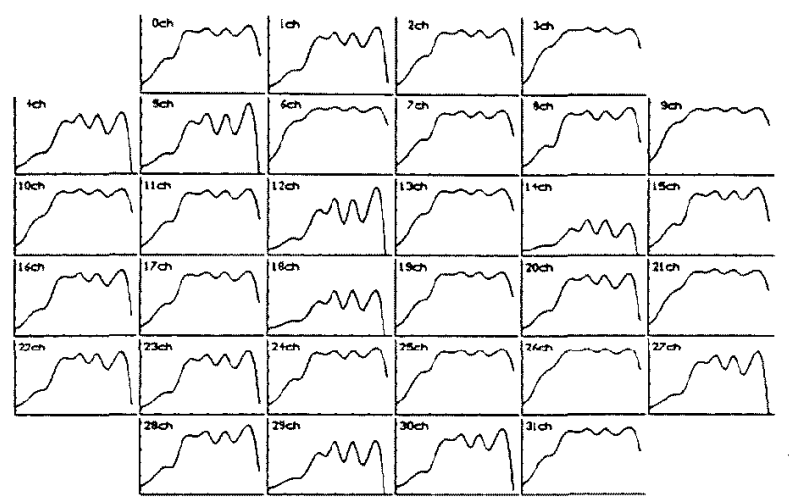

Fig. 4 Spectrum data from 32-ch POF sensor array.

\section{Results and Discussion}

\subsection{Multichannel Spectra Measurement}

Figure 3 shows a typical set of spectrum data $(V \sim \lambda)$ from five POF sensors with the same dye coating. The smooth spectrum curves were obtained by spline interpolation of the discrete intensity values ( $V$ ) at the wavelengths corresponding to the 13 band pass filters. Figure 4 show a set of spectrum data $(V \sim \lambda)$ from a $32-c h$ POF sensor array in air. The 32 spectra were simultaneously measured by changing the bandpass filter in Fig. 2. From Fig. 3 and Fig. 4 , it is seen that there is large difference in spectrum intensity between channels. The use of a normalized valiable, absorbance $\Delta A=\log \left(V_{0} / V\right)$, has been found to decrease the errors resulting from the difference. In the first stage of our work, the absorbance spectrum data had poor reproducibility as shown in Fig. 5 (top). After careful investigation, the poor reproducibility was found to be due to the variation in positions of the band-pass filter and the fibers in the optical axis when the filter was changed by hand-operation. Thus, the poor reproducibility was improved as shown in Fig. 5 (bottom) by
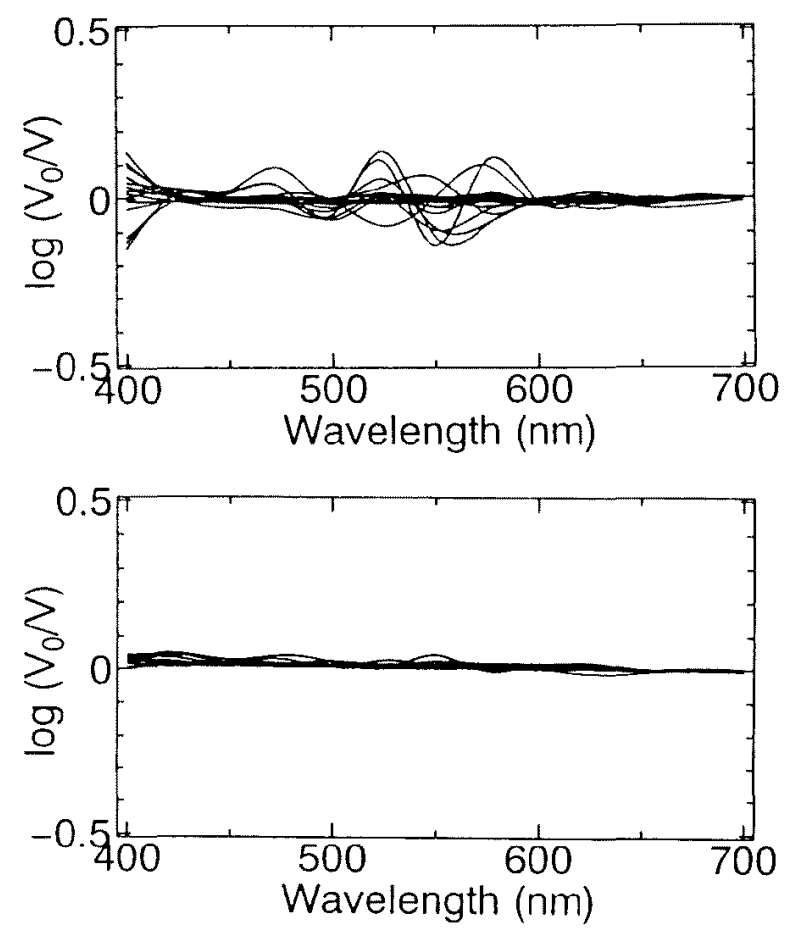

Fig. 5 Improved reproducibility of $\log \left(V_{0} / N\right) \sim \lambda$, before (top) and after (bottom) investigations.

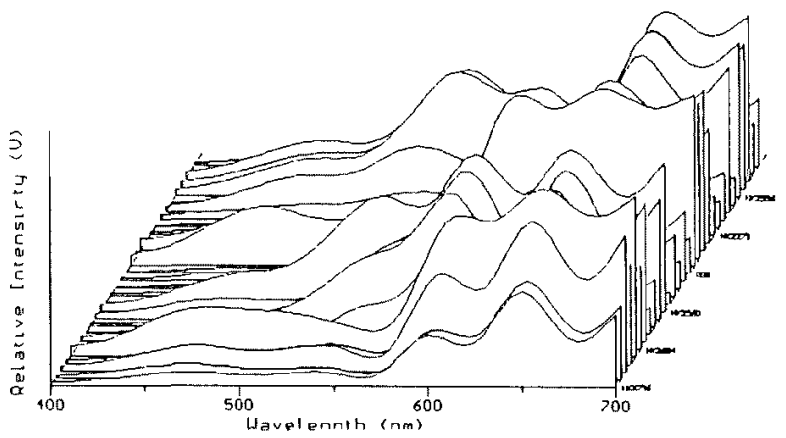

Fig. 6 Spectra set from 32-ch P0F sensor array.

careful arrangement of the optical fiber bandle and careful filter-change operation.

\subsection{Gas Discrimination Experiments Using ANN}

Figure 6 shows a set of 32 spectra data from the 32-ch POF sensor array with six different dye coatings used. Figures 7 and 8 show typical sets of absorbance spectra $\log \left(V_{0} / V\right) \sim \lambda$ from the 32-ch POF sensor array in air (Fig. 7), and in methanol gas(Fig. 8). The 32 spectra data set (13 $\times 32=416)$ were used as the ANN input data set for gas discrimination.

Figure 9 shows the ANN structure used for discrimination of alcoholic gases. A simple 
three-layer back-propagation neural network was used. The network was trained to a root mean square error of 0.01 using back-propagation algorithm. The unit numbers of input layer, hidden layer, and output layer were $426(=13 \times 32)$, 20 , and 4 .

Table 1 shows the discrimination results of alcoholic gases: methano1, ethano1, 1-propanol, 2-propanol. Apparently, the four alcohol gases having similar chemical structure can be discriminated by this $32-\mathrm{ch}$ POF sensor system, but not by the semiconductor gas sensor system ${ }^{(6)}$.

In Table 1, it is seen that the increase in wavelength point from 1 to $13, \mathrm{i}, \mathrm{e}$, in input unit from 32 to 416 leads to an improvement in recognition rate. The recognation rate using 13 wavelength points was higher (100\% except ethano1) than those using three wavelength points ( $3 \times 32=96$ inputs) and one wavelength point (1 $132=32$ inputs).

The increase in sensor number from $6^{(4)}$ to 32 , i. e., in ANN inputs from $78^{(4)}$ to 416 contributes to an improvement in the recognition rate. The recognition rates of methanol and ethanol were $12 / 12$ and $10 / 12$ in 416 inputs (Table 1).

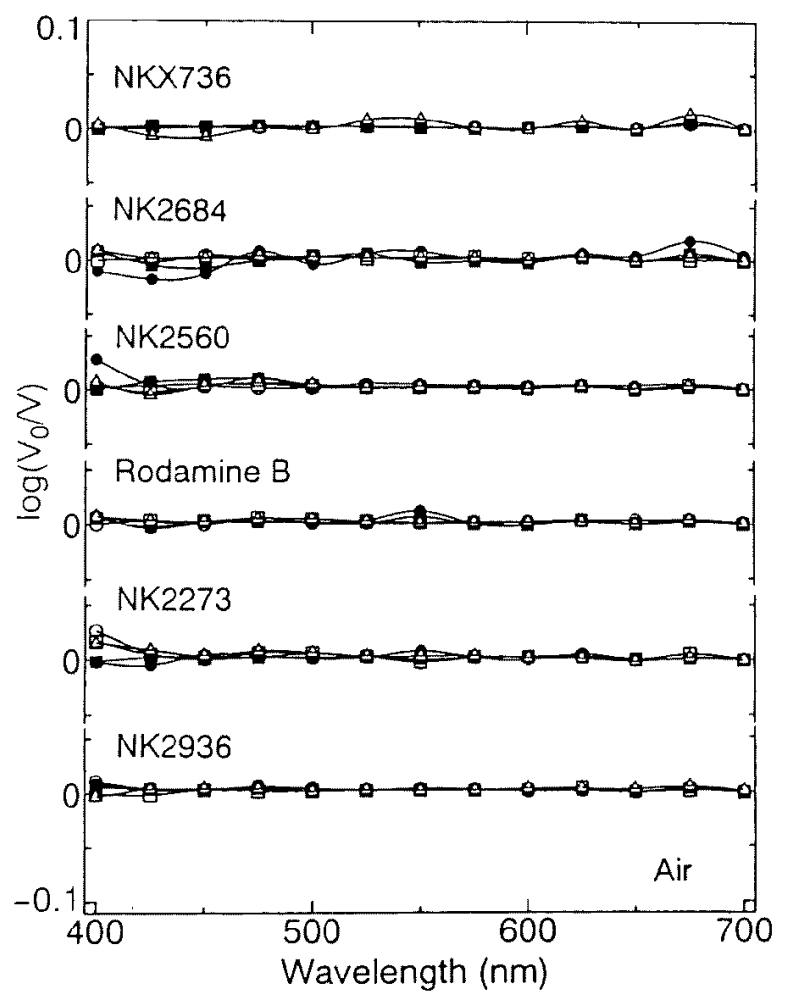

Fig. 7 Absorbance spectrum data from the 32 -ch $\mathrm{POF}$ sensor array in air.
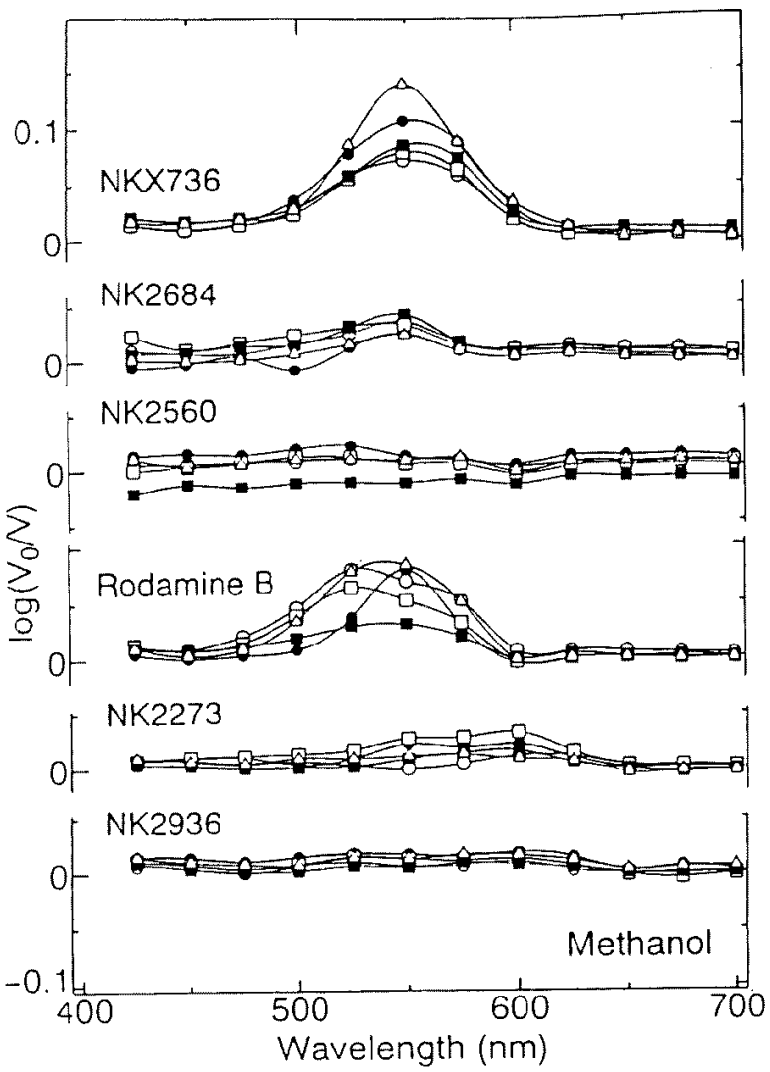

Fig. 8 Absorbance spectrum data from the 32-ch $\mathrm{POF}$ sensor array in methanol gas.

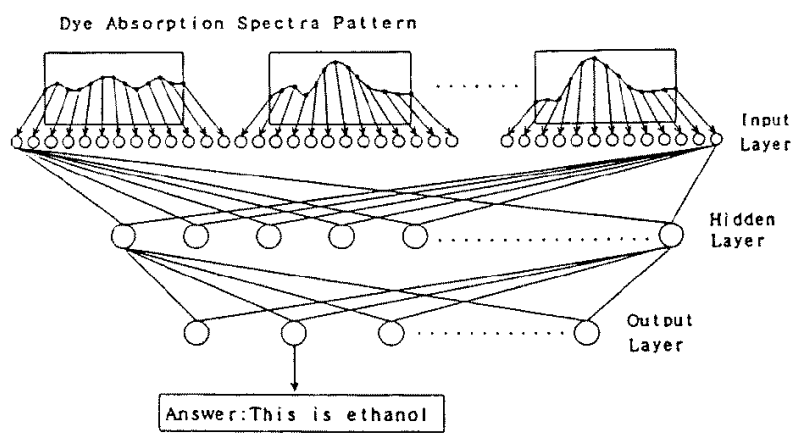

Fig. 9 ANN structure used for gas discrimination.

Table 1 Discrimination results of alcoholic gases

\begin{tabular}{|c|c|c|c|}
\hline gas & & recognition rate & \\
\hline & $\begin{array}{l}\text { one point } \\
\text { at } 474 \mathrm{~nm}\end{array}$ & $\begin{array}{l}3 \text { points at } 474, \\
525 \text {, and } 598 \mathrm{~nm}\end{array}$ & $\begin{array}{l}13 \text { points } \\
400-700 \mathrm{nI}\end{array}$ \\
\hline methanol & $7 / 12$ & $12 / 12$ & $12 / 12$ \\
\hline ethanol & $5 / 12$ & $7 / 12$ & $10 / 12$ \\
\hline 1-propanol & $9 / 12$ & $9 / 12$ & $12 / 12$ \\
\hline 2-propanol & $9 / 12$ & $9 / 12$ & $12 / 12$ \\
\hline
\end{tabular}


In the other hand, they were $10 / 12$ and $9 / 12$ in 78 inputs $^{(4)}$. The former system (418 inputs) discriminated between 1-propanol and 2-propanol, but the latter system ${ }^{(4)}$ (78 inputs) could not discriminate the two similar gases.

This improvement by the increase in sensor number depends probably on the dye coating and the gas tested. A more deetailed investigation is required on the effect on gas discrimination of the increase in sensor number.

The author would like to thank K. Takatuka and M. Nishimura for their support of the experimental work. This work was supported in part by a Grant-in-Aid for Scientific Research from the Ministry of Education, Science and Culture. (Manuscript received March 29, 1996, revised Sep. 19, 1996)

\section{References}

(1) T. Nakamoto, K. Fukunishi and T. Moriizumi: "Identification Capability of 0dor Sensor Using Quartz-resonator Array and Neura1network Pattern Recognition, Sensors and Actuators. B1, 473-476(1990).

(2) J. M. Slater, J. Paynter and E. J. Watt: "Multilayer Conducting Polymer Gas Sensor Arrays for 01factory Sensing, Analyst, 118. 379-384(1993).

(3) S. Yamakawa: "Optica1 Odor Sensors Using Potential-Sensitive Dye Coatings, Trans. IEICE, J76-CII, 571-575(1993); Electronics and Communications in Japan, Part2, 76(12), 110-115(1993).

(4) S. Yamakawa and M. Nishimura: "Organic Gas Discrimination by Dye Spectrum Pattern Recognition Using Fiber-Optic Sensor Array and Artificial Newral Network, Proc. 36th Japan Joint Automatic Control Conference, 363-364(1993. 10).

(5) K. Mori: "A System for Recognition of Odor Molecules", J. IEICE, 79, 1209-1215(1993).

(6) S. Yamakawa and A. Yamaguchi: "Odor Pattern Recognition Using Gas Sensor Array and $\mathrm{Neu}^{-}$ ral Network, Trans. IEE Japan, C113, 621-626 (1993).
Shinzo Yamakawa (Member) He received a B.S. from Kanazawa University in

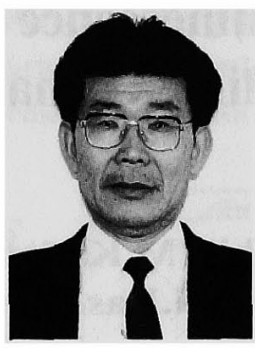
1962 and a M. S. and a Ph. D from Kyoto University in 1966 and 1980. He joined NTT in 1966 and was involved in research \& development of optical fiber fabrication and maerial technology. He retired from NTT in 1987 and became a Professor of Department of Computer Engineering, Toyama National College of Maritime Technology. He has been involved in research on fiber-optic sensors. He is a member of IEEJ, JJAP, IEICE, SICE and IEEE. 